Vol. 27, Issue 5, October 2004

Editorial

437 'Retarded Pharmaceuticals' Assuming a Clinical Role Muggia, F.M. (New York)

Original Articles

441 Phase II Study of Single-Agent Pegylated Liposomal Doxorubicin HCI (PLD) in Metastatic Breast Cancer After First-Line Treatment Failure

Mlineritsch, B.; Mayer, P.; Rass, C.; Reiter, E.; Russ, G.; Vesenmayer, G. (Salzburg); Oberaigner, W. (Innsbruck); Hausmaninger, H. (Salzburg)

449 Second-Line Treatment of Advanced Colorectal Cancer with a Weekly Simultaneous 24-Hour Infusion of 5-Fluorouracil and Sodium-Folinate: A Multicentre Phase II Trial

Kuhfahl, J.; Steinbrecher, C. (Regensburg); Wagner, T. (Lübeck); Wagner, H. (Weiden); Fritze, D. (Darmstadt); Link, H. (Kaiserslautern); Schulte, F.; Pichlmeier, U. (Wedel); Kreuser, E.-D. (Regensburg)

457 Relationship between Psychosocial Distress, Treatment Need, and Use of Psychotherapeutic Interventions within a Psychosomatic Liaison Service in Hematological Oncology Fritsche, K.; Struss, Y.; Hammel, A.; Bertz, H.; Stein, B. (Freiburg i.Br.)

462 Impact of Chemoradiotherapy-Induced Anemia on Survival in Uniformly Staged Patients with Locally Advanced Squamous Cell Carcinoma of the Esophagus Hofheinz, R.-D.; Raab, B.; Mai, S.; Wenz, F.; Willeke, F.; Emig, M.; Buchheidt, D.; Hehlmann, R.; Hochhaus, A. (Mannheim)

\section{Casuistic Contributions}

469 Bilateral Metastatic Rhabdomyosarcoma to the Breast in an Adolescent Female: Touch Imprint Cytology and Implication of MyoD1 Nuclear Antigen

Tamiolakis, D. (Chania); Venizelos, I. (Salonica); Nikolaidou, S. (Chania); Prassopoulos, P.; Alexiadis, G.; Simopoulos, C.; Papadopoulos, N. (Alexandroupolis)
Band 27, Heft 5, Oktober 2004

Editorial

437 Pegylierte Therapeutika in der klinischen Praxis Muggia, F.M. (New York)

Originalarbeiten

441 Phase-II-Studie zur Bewertung von Caelyx ${ }^{\circledR}$. Monotherapie (pegyliertes liposomales Doxorubicin$\mathrm{HCl}$ ) beim metastasierten Mammakarzinom nach Versagen einer First-Line Therapie Mlineritsch, B.; Mayer, P.; Rass, C.; Reiter, E.; Russ, G.; Vesenmayer, G. (Salzburg); Oberaigner, W. (Innsbruck); Hausmaninger, H. (Salzburg)

449 Zweitlinientherapie fortgeschrittener kolorektaler Karzinome mit einer wöchentlichen 24-StundenInfusion von 5-Fluorouracil und Natriumfolinat: Eine multizentrische Phase-II-Studie

Kuhfahl, J.; Steinbrecher, C. (Regensburg); Wagner, T. (Lübeck); Wagner, H. (Weiden); Fritze, D. (Darmstadt); Link, H. (Kaiserslautern); Schulte, F.; Pichlmeier, U. (Wedel); Kreuser, E.-D. (Regensburg)

457 Zusammenhang zwischen psychosozialen Belastungen, Behandlungsbedarf und Inanspruchnahme von psychotherapeutischen Leistungen eines psychosomatischen Liaisondienstes in der hämatologischen Onkologie Fritsche, K.; Struss, Y.; Hammel, A.; Bertz, H.; Stein, B. (Freiburg i.Br.)

462 Einfluss der Anämie auf das Überleben bei Patienten mit einheitlich diagnostiziertem lokal fortgeschrittenem Plattenepithelkarzinom des Ösophagus Hofheinz, R.-D.; Raab, B.; Mai, S.; Wenz, F.; Willeke, F.; Emig, M.; Buchheidt, D.; Hehlmann, R.; Hochhaus, A. (Mannheim)

Kasuistiken

469 Bilaterale Brustmetastasen eines Rhabdomyosarkoms bei einem heranwachsenden Mädchen: Zytologische Untersuchung von Abklatschpräparaten und Bedeutung des nuklearen Antigens MyoD1

Tamiolakis, D. (Chania); Venizelos, I. (Salonica); Nikolaidou, S. (Chania); Prassopoulos, P.; Alexiadis, G.; Simopoulos, C.; Papadopoulos, N. (Alexandroupolis)

\begin{tabular}{ll}
\hline KARGER & @ 2004 S. Karger GmbH, Freiburg \\
Fax +497614520714 & Artikel (Volltext) und Inhaltsverzeichnisse \\
$\begin{array}{l}\text { E-mail Information@Karger.de } \\
\text { www.karger.com }\end{array}$ & sowie das vorläufige Inhaltsverzeichnis des nächsten Heftes: \\
www.karger.com/onk_bk.htm
\end{tabular}


Vol. 27, Issue 5, October 2004

472 Severe Pulmonary Hypertension in Chronic Idiopathic Myelofibrosis

Halank, M.; Marx, C.; Baretton, G. (Dresden); Müller, K.-M. (Bochum); Ehninger, G.; Höffken, G. (Dresden)

477 Breast Cancer in a Male Patient after Treatment of Acute Lymphoblastic Leukemia Including Total Body Irradiation and Bone Marrow Transplantation Latz, D.; Alfrink, M.; Nassar, N. (Coburg); Beyerle, C. (Kronach)

480 Panhypopituarism in a Patient with Breast Cancer Sturm, I.; Kirschke, S.; Krahl, D.; Dörken, B. (Berlin)

\section{Review Articles}

487 The Innate Immune Response in the Central Nervous System and Its Role in Glioma Immune Surveillance

Friese, M.A.; Steinle, A.; Weller, M. (Tübingen)

492 Primary Extracutaneous Malignant Melanoma: A Comprehensive Review with Emphasis on Treatment Thoelke, A. (Mannheim); Willrodt, S.; Hauschild, A. (Kiel); Schadendorf, D. (Mannheim)

500 Chemotherapy in Elderly Patients with Advanced Lung Cancer. Part I: General Aspects and Treatment of Small Cell Lung Cancer (SCLC)

Honecker, F. (Tübingen); Wedding, U. (Jena); Bokemeyer, C. (Tübingen) for the Interdisziplinäre Arbeitsgruppe Geriatrische Onkologie der DGHO/DGG

506 Treatment of Locally Recurrent Rectal Cancer with Special Focus on Regional Pelvic Hyperthermia Hildebrandt, B.; Wust, P.; Gellermann, J.; Nicolaou, A.; Trappe, R.-U.; Felix, R.; Riess, H.; Rau, B. (Berlin)
Band 27, Heft 5, Oktober 2004

472 Schwere pulmonale Hypertonie bei chronisch idiopathischer Myelofibrose

Halank, M.; Marx, C.; Baretton, G. (Dresden); Müller, K.-M. (Bochum); Ehninger, G.; Höffken, G. (Dresden)

477 Entwicklung eines Mammakarzinoms bei einem männlichen Patienten nach der Behandlung einer akuten lymphoblastischen Leukämie mit Ganzkörperbestrahlung und Knochenmarktransplantation

Latz, D.; Alfrink, M.; Nassar, N. (Coburg); Beyerle, C. (Kronach)

480 Panhypopituitarismus bei einer Patientin mit Mammakarzinom

Sturm, I.; Kirschke, S.; Krahl, D.; Dörken, B. (Berlin)

Übersichtsarbeiten

487 Die angeborene Immunität im zentralen Nervensystem und ihre Rolle bei der Immunüberwachung von Gliomen Friese, M.A.; Steinle, A.; Weller, M. (Tübingen)

492 Primär extrakutane Maligne Melanome: Eine umfassende Übersicht unter Berïcksichtigung der Therapie Thoelke, A. (Mannheim); Willrodt, S.; Hauschild, A. (Kiel); Schadendorf, D. (Mannheim)

500 Chemotherapie in der Behandlung älterer Patienten mit fortgeschrittenem Bronchialkarzinom. Teil I: Allgemeine Einfuihrung und Behandlung von Patienten mit kleinzelligem Bronchialkarzinom (SCLC) Honecker, F. (Tübingen); Wedding, U. (Jena); Bokemeyer, C. (Tübingen) for the Interdisziplinäre Arbeitsgruppe Geriatrische Onkologie der DGHO/DGG

506 Behandlung des lokal rezidivierten Rektumkarzinoms unter besonderer Berücksichtigung der regionalen Radiofrequenz-Hyperthermie Hildebrandt, B.; Wust, P.; Gellermann, J.; Nicolaou, A.; Trappe, R.-U.; Felix, R.; Riess, H.; Rau, B. (Berlin)

\begin{tabular}{ll}
\hline KARGER & @ 2004 S. Karger GmbH, Freiburg \\
Fax +49 76145207 14 & Artikel (Volltext) und Inhaltsverzeichnisse \\
$\begin{array}{l}\text { E-mail Information@Karger.de } \\
\text { www.karger.com }\end{array}$ & sowie das vorläufige Inhaltsverzeichnis des nächsten Heftes: \\
www.karger.com/onk_bk.htm
\end{tabular}


Vol. 27, Issue 5, October 2004

512 Book Reviews

514 PharmaForum

516 PharmaNews

516 PharmaTicker

518 Meetings and Conferences

505 Imprint

Prospects for contents of the next issues are given on page 520.
Band 27, Heft 5, Oktober 2004

512 Buchbesprechungen

514 PharmaForum

516 PharmaNews

516 PharmaTicker

518 Tagungen und Kongresse

505 Impressum

Einen Ausblick auf den Inhalt der kommenden Hefte finden Sie auf Seite 520.

\section{KARGER}

Fax +497614520714

E-mail Information@Karger.de

www.karger.com

\section{(C) 2004 S. Karger GmbH, Freiburg}

Artikel (Volltext) und Inhaltsverzeichnisse

sowie das vorläufige Inhaltsverzeichnis des nächsten Heftes: www.karger.com/onk_bk.htm 